Bull. Austral. Math. Soc.

$05 \mathrm{C} 50,15 \mathrm{~A} 18$

VOL. 69 (2004) [133-136]

\title{
CHARACTERISTIC POLYNOMIALS OF GRAPH COVERINGS
}

\author{
Rongquan Feng, Jin Ho KWak and Jaeun Lee
}

In this note, a formula for the characteristic polynomial of any (regular or irregular) graph covering is described.

Let $G$ be a finite simple graph with vertex set $V(G)=\left\{v_{1}, v_{2} \ldots, v_{m}\right\}$. The adjacency matrix $A(G)=\left(a_{i j}\right)$ is the $m \times m$ matrix with $a_{i j}=1$ if $v_{i}$ and $v_{j}$ are adjacent and $a_{i j}=0$ otherwise. The characteristic polynomial of $G$, denoted by $\Phi(G ; \lambda)$, is the characteristic polynomial $\operatorname{det}(\lambda I-A(G))$ of $A(G)$.

A covering projection (or simply covering) from a graph $\tilde{G}$ to another $G$ is a surjection $p: V(\widetilde{G}) \rightarrow V(G)$ such that $\left.p\right|_{N(\tilde{v})}: N(\widetilde{v}) \rightarrow N(v)$ is a bijection for all vertices $v \in V(G)$ and $\tilde{v} \in p^{-1}(v)$, where $N(v)$, the neighbourhood of $v$, is the set of vertices adjacent to $v$. Sometimes, a graph $\widetilde{G}$ is also called a covering of $G$ with the projection $p: \widetilde{G} \rightarrow G$, and it is $n$-fold if $p$ is $n$-to-one.

Every edge of a graph $G$ gives rise to a pair of oppositely directed edges. By $e^{-1}=v u$, we mean the reverse directed edge to a directed edge $e=u v$. A directed edge is also called an $\operatorname{arc}$ and the set of arcs of the graph $G$ is denoted by $D(G)$. Let $S_{n}$ be the symmetric group on $\Omega=\{1,2, \ldots, n\}$. A voltage assignment $\phi$ of $G$ is a function $\phi: D(G) \rightarrow S_{n}$ with the property that $\phi\left(e^{-1}\right)=\phi(e)^{-1}$ for each $e \in D(G)$. The derived graph $G^{\phi}$ from a voltage assignment $\phi$ is defined as $V\left(G^{\phi}\right)=V(G) \times \Omega$, and $(u, i)$ and $(v, j)$ are adjacent if $u v \in D(G)$ and $j=i^{\phi(u v)}$. The first coordinate projection $p_{\phi}: G^{\phi} \rightarrow G$ is an $n$-fold covering. Let $C^{1}(G ; n)$ denote the set of all voltage assignments $\phi: D(G) \rightarrow S_{n}$ of $G$. Gross and Tucker [2] showed that every $n$-fold covering $\widetilde{G}$ of a graph $G$ can be derived from a voltage assignment in $C^{\mathrm{l}}(G ; n)$.

Characteristic polynomials of some graph coverings have already been computed. Chae, Kwak and Lee [1] have done it for double coverings of a graph. The characteristic polynomial of a graph covering when its voltages lie in an Abelian group or in a dihedral group was computed by Kwak and others [3, 4]. Mizuno and Sato [5] gave a formula for the characteristic polynomial of a regular covering. In this note, a formula for the characteristic polynomial of any (regular or irregular) graph covering is described, as an extension of all of the previous works.

Received 5th August, 2003

The first author is supported by NSF of China (No. 10001005) and the last two are supported by $\mathrm{Com}^{2} \mathrm{MaC}-\mathrm{KOSEF}$ in Korea.

Copyright Clearance Centre, Inc. Serial-fee code: 0004-9727/04 \$A2.00+0.00. 
Let $\vec{G}$ denote the digraph obtained from $G$ by replacing each edge of $G$ with a pair of oppositely directed edges and let $\phi \in C^{1}(G, n)$. For each $\gamma \in S_{n}$, let $\vec{G}_{(\phi, \gamma)}$ denote the spanning subgraph of the digraph $\vec{G}$ whose directed edge set is $\phi^{-1}(\gamma)$. Let $V(G)=\left\{v_{1}, v_{2}, \ldots, v_{m}\right\}$ again. We define an order relation $\leqslant$ on $V\left(G^{\phi}\right)$ as follows: for $\left(v_{i}, s\right),\left(v_{j}, t\right) \in V\left(G^{\phi}\right),\left(v_{i}, s\right) \leqslant\left(v_{j}, t\right)$ if and only if either $s<t$ or $s=t$ and $i \leqslant j$. Let $P(\gamma)$ denote the $n \times n$ permutation matrix associated with $\gamma \in S_{n}$, that is, its $(s, t)$-entry $P(\gamma)_{s t}=1$ if $s^{\gamma}=t$ and $P(\gamma)_{s t}=0$ otherwise. The tensor product $A \otimes B$ of the matrices $A$ and $B$ is considered as the matrix $B$ having the element $b_{s t}$ replaced by the matrix $A b_{s t}$. Kwak and Lee ([3]) expressed the adjacency matrix $A\left(G^{\phi}\right)$ of a graph covering $G^{\phi}$ as

$$
A\left(G^{\phi}\right)=\sum_{\gamma \in S_{n}} A\left(\vec{G}_{(\phi, \gamma)}\right) \otimes P(\gamma) .
$$

Let $\Gamma$ be a finite group. A representation $\rho$ of a group $\Gamma$ over the complex field $\mathbb{C}$ is a group homomorphism from $\Gamma$ to the general linear group $\mathrm{GL}(r, \mathbb{C})$ of invertible $r \times r$ matrices over $\mathbb{C}$. The number $r$ is called the degree of the representation $\rho$ (see [6]). Suppose that $\Gamma \leqslant S_{n}$ is a permutation group on $\Omega$. It is clear that $P: \Gamma \rightarrow \mathrm{GL}(r, \mathbb{C})$ defined by $\gamma \rightarrow P(\gamma)$, where $P(\gamma)$ is the permutation matrix associated with $\gamma \in \Gamma$ corresponding to the action of $\Gamma$ on $\Omega$, is a representation of $\Gamma$. It is called the permutation representation. Let $\rho_{1}=1, \rho_{2}, \ldots, \rho_{\ell}$ be the irreducible representations of $\Gamma$ and let $f_{i}$ be the degree of $\rho_{i}$ for each $1 \leqslant i \leqslant \ell$, where $f_{1}=1$ and $\sum_{i=1}^{\ell} f_{i}^{2}=|\Gamma|$. It is well-known [6] that the permutation representation $P$ can be decomposed as the direct sum of irreducible representations. In other words, there exists an invertible matrix $M$ such that

$$
M^{-1} P(\gamma) M=\bigoplus_{i=1}^{\ell}\left(\rho_{i}(\gamma) \otimes I_{m_{i}}\right)
$$

for any $\gamma \in \Gamma$, where $m_{i} \geqslant 0$ is the multiplicity of the irreducible representation $\rho_{i}$ in the permutation representation $P$ and $\sum_{i=1}^{\ell} m_{i} f_{i}=n$. Notice that $m_{1}$ is the number of orbits under the action of the group $\Gamma$ on $\Omega$. So $m_{1} \geqslant 1$.

Now let $\phi \in C^{1}(G, n)$ and $\Gamma=\langle\phi(e) \mid e \in D(G)\rangle$, the subgroup generated by the voltages $\phi(e)$. Noting that $\sum_{i=1}^{\ell} m_{i} f_{i}=n$, from equations (1) and (2) we have

$$
\left(I_{m} \otimes M\right)^{-1}\left(\lambda I_{m n}-A\left(G^{\phi}\right)\right)\left(I_{m} \otimes M\right)=\bigoplus_{i=1}^{\ell}\left[\left(\lambda I_{m f_{i}}-\sum_{\gamma \in \Gamma} A\left(\vec{G}_{(\phi, \gamma)}\right) \otimes \rho_{i}(\gamma)\right) \otimes I_{m_{i}}\right] .
$$

Since $\rho_{1}(\gamma)=1$ for any $\gamma \in \Gamma$ and $A(G)=\sum_{\gamma \in \Gamma} A\left(\vec{G}_{(\phi, \gamma)}\right)$, we get

$$
\Phi\left(G^{\phi} ; \lambda\right)=\Phi(G ; \lambda)^{m_{1}} \prod_{i=2}^{\ell}\left[\operatorname{det}\left(\lambda I_{m f_{i}}-\sum_{\gamma \in \Gamma} A\left(\vec{G}_{(\phi, \gamma)}\right) \otimes \rho_{i}(\gamma)\right)\right]^{m_{i}} .
$$


Summarising our discussions, we have the following theorem.

MaIN Theorem. Let $G$ be a graph with $m$ vertices, $\phi \in C^{1}(G, n)$ a voltage assignment on $G$ and $\Gamma=\langle\phi(e) \mid e \in D(G)\rangle$. Let $\rho_{1}=1, \rho_{2}, \ldots, \rho_{\ell}$ be the irreducible representations of $\Gamma$ and let $f_{i}$ be the degree of $\rho_{i}$ for each $1 \leqslant i \leqslant \ell$ with $f_{1}=1$. Then the characteristic polynomial of the $n$-fold covering $G^{\phi}$ of $G$ derived from the voltage assignment $\phi$ is

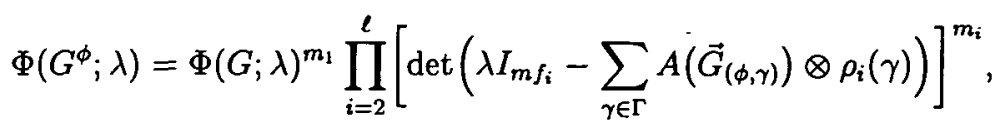

where $m_{i}$ is the multiplicity of $\rho_{i}$ in the permutation representation $P$ of $\Gamma$.

Since $m_{1} \geqslant 1$, it gives that for every covering graph $G^{\phi}$ of the graph $G$, the characteristic polynomial $\Phi(G ; \lambda)$ is a divisor of the characteristic polynomial $\Phi\left(G^{\phi} ; \lambda\right)$, in $[\mathbf{1}$, Corollary 1]. When $\Gamma$ is a regular subgroup of $S_{n}$, the permutation representation $P$ of $\Gamma$ is equivalent to the (right) regular representation and the covering $G^{\phi}$ is a regular covering of $G$. In this case, each multiplicity $m_{i}$ is equal to $f_{i}$, the degree of the irreducible representation $\rho_{i}$. Therefore, Mizuno and Sato's [5, Theorem 2] can be derived from the main theorem. Furthermore, When $\Gamma$ is Abelian or $\Gamma$ is the dihedral group of order $2 n$, the same results as in [3] and in [4] can also be deduced.

We close this note by giving a computational example which could not be done by using any formula that was known before. Let $G$ be any graph, $\phi \in C^{1}(G, 4)$ a voltage assignment on $G$ and $\Gamma=\langle\phi(e) \mid e \in D(G)\rangle=S_{4}$. Note that the symmetric group $S_{4}$ can be generated by (12) and (1234). Then, the permutation representation $P$ of $S_{4}$ can be decomposed by $P=\rho_{1} \oplus \rho_{2}$, where $\rho_{1}=1$, the trivial representation, and $\rho_{2}$ is defined on the generators of $\Gamma$ by

$$
\rho_{2}((12))=\left[\begin{array}{lll}
0 & 1 & 0 \\
1 & 0 & 0 \\
0 & 0 & 1
\end{array}\right] \text { and } \rho_{2}((1234))=\left[\begin{array}{rrr}
-1 & 0 & 0 \\
0 & 0 & -1 \\
0 & 1 & 0
\end{array}\right] .
$$

Therefore, the characteristic polynomial of the 4-fold covering $G^{\phi}$ of $G$ derived from the voltage assignment $\phi$ is

$$
\Phi\left(G^{\phi} ; \lambda\right)=\Phi(G ; \lambda) \operatorname{det}\left(\lambda I_{3|V(G)|}-\sum_{\gamma \in S_{4}} A\left(\vec{G}_{(\phi, \gamma)}\right) \otimes \rho_{2}(\gamma)\right) .
$$

For example, for the diamond graph $G$ which is the complete graph $K_{4}$ minus an edge, one can see that

$$
\Phi(G, \lambda)=\lambda(\lambda+1)\left(\lambda^{2}-\lambda-4\right) .
$$


Consider a voltage assignment $\phi$ which is defined as in Figure 1.

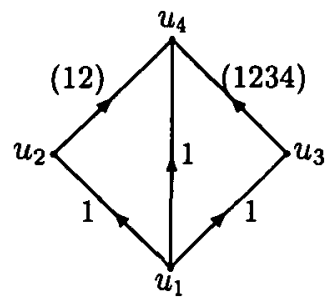

Figure 1: An $S_{4}$-voltage assignment $\phi$ on the diamond graph

From equation (3), one can get the characteristic polynomial of the graph $G^{\phi}$ as

$\Phi\left(G^{\phi} ; \lambda\right)=\Phi(G ; \lambda) \lambda^{2}\left(\lambda^{10}-12 \lambda^{8}+2 \lambda^{7}+51 \lambda^{6}-22 \lambda^{5}-87 \lambda^{4}+66 \lambda^{3}+39 \lambda^{2}-54 \lambda+12\right)$.

\section{REFERENCES}

[1] Y. Chae, J.H. Kwak and J. Lee, 'Characteristic polynomials of some graph bundles', $J$. Korean Math. Soc. 30 (1993), 229-249.

[2] J.L. Gross and T.W. Tucker, 'Generating all graph coverings by permutation voltage assignments', Discrete Math. 18 (1977), 273-283.

[3] J.H. Kwak and J. Lee, 'Characteristic polynomials of some graph bundles $\mathbb{I}$ ', Linear and Multilinear Algebra 32 (1992), 61-73.

[4] J.H. Kwak and Y.S. Kwon, 'Characteristic polynomials of graph bundles having voltages in a dihedral group', Linear Algebra Appl. 336 (2001), 99-118.

[5] H. Mizuno and I. Sato, 'Characteristic polynomials of some graph coverings', Discrete Math. 142 (1995), 295-298.

[6] B.E. Sagan, The Symmetric group, (2nd edition) (Springer-Verlag, New York, 2001).

LMAM, School of Mathematical Sciences

Peking University

Beijing 100871

Peoples Republic of China

e-mail: fengrq@math.pku.edu.cn
Department of Mathematics

Pohang University of Science and Technology

Pohang, 790-784

Korea

e-mail: jinkwak@postech.ac.kr

Department of Mathematics

Yeungnam University

Kyongsan, 712-749

Korea

e-mail: julee@yu.ac.kr 